\title{
Trip Cost Estimation of Connected Autonomous Vehicle Mixed Traffic Flow in a Two-Route Traffic Network
}

\author{
Zhizhen Liu (D), Hong Chen $\left(\mathbb{D}\right.$, Hengrui Chen $\mathbb{D}^{D}$, Xiaoke Sun, and Qi Zhang \\ College of Transportation Engineering, Chang'an University, Xi'an 710000, China \\ Correspondence should be addressed to Hong Chen; glch@chd.edu.cn
}

Received 17 June 2020; Revised 8 September 2020; Accepted 25 September 2020; Published 10 October 2020

Academic Editor: Kun Wang

Copyright (c) 2020 Zhizhen Liu et al. This is an open access article distributed under the Creative Commons Attribution License, which permits unrestricted use, distribution, and reproduction in any medium, provided the original work is properly cited.

\begin{abstract}
With the advancement of connected autonomous vehicle (CAV) technology, research on future traffic conditions after the popularization of CAVs needs to be resolved urgently. Bounded rationality of human drivers is essential for simulating traffic flow precisely, but few studies focus on the traffic flow simulation considered bounded rationality in CAV mixed traffic flow. In this study, we introduce random bounded rationality into the hybrid feedback strategy (HFS) under CAV mixed traffic flow to explore the impacts of CAV penetration rate on the trip cost of vehicles. First, we investigated the bounded rationality of drivers, and we found that it follows normal contribution. Then, we proposed HFS considering random bounded rationality and the CAV penetration rate to simulate the traffic condition. The numerical results show that the enhancement of the CAV penetration rate could reduce total trip cost. The research could help us to simulate the CAVs mixed traffic flow more precisely and realistically.
\end{abstract}

\section{Introduction}

Along with the development of the Internet of vehicle technology and the autonomous vehicle technology, mixed traffic conditions of human-driven vehicles (HDVs) and connected autonomous vehicles (CAVs) should be studied $[1,2]$. Simulation of the mixed traffic condition is essential for addressing the future development of intelligent transportation systems.

In recent years, researchers have proposed many traffic flow models in previous studies. Traffic flow models could be generally divided into macromodels [3-5] and micromodels [6-9]. Macromodels aim to study the relationship between flow, speed, and density. In contrast, micromodels focused on driving behaviors. However, the researches above did not study the traffic flow models based on the mixed traffic conditions of HDVs and CAVs. Most studies focused on the traffic flow models of HDVs. Many researchers proposed car-following models to simulate the traffic flow based on HDVs, but they ignored the impacts of humans' bounded rationality on the traffic flow operation. Therefore, Ou et al. [10] incorporated bounded rationality into two feedback strategies to study vehicle trip cost under the feedback strategies. Moreover, several researchers focused on carfollowing models based on CAVs.

However, the researches above did not study the traffic flow model considering the mixed traffic conditions of HDVs and CAVs, and their feedback strategies ignored the penetration rate of CAVs. Furthermore, they did not consider the randomness of HDV drivers' bounded rationality. However, under the background of the popularity of CAVs in the future, the phenomenon of mixed traffic between CAVs and HDVs will inevitably appear. Therefore, the study of mixed traffic flow between CAVs and HDVs is imperative.

To solve the problem, we propose a hybrid feedback strategy (HFS) considering the penetration rate of CAVs and random bounded rationality. Then, we explore the regulation of random bounded rationality of HDV drivers based on the questionnaire. Next, we study the impacts of the penetration rate of CAVs on each vehicle's travel time in the two-route traffic network. Additionally, in this study, our basic hypotheses are as follows: (1) HDVs and CAVs randomly pass the two-route traffic network; (2) two routes of the two-route traffic network are homogeneous; (3) CAVs make behavioral choices under absolutely rational circumstances. 
This paper has three main contributions: (1) we fit the distribution of values of random bounded rationality based on investigations; (2) the proposed HFS considered the randomness of drivers' bounded rationality and the CAV penetration rate to simulate the traffic flow; (3) we explore the impacts of the CAV penetration rate on the trip cost in a two-route traffic network. Furthermore, the research is valuable for promoting the use of CAVs in the future, and it can help us solve the route guidance problem in the context of the mixed traffic flow between CAVs and HDVs.

This paper is organized as follows: literature review is included in Section 2, and Section 3 describes the data we used in this study. The method is indicated in Section 4, and the results of the case study are included in Section 5. Finally, Section 6 contains the discussion, the conclusion, and future research.

\section{Literature Review}

2.1. Car-Following Model of HDVs. Many studies focused on the microtraffic flow models of HDVs. In terms of carfollowing models, several researchers improved the methods. These researches could be divided into two types: (1) calibrating the parameters of existing car-following models; (2) learning a new car-following model from the historical trajectory data based on the machine learning methods.

The first kind of researches aims to calibrate the parameters of existing car-following models. The studies generally test the macroscopic features consistency between simulation result and physical truth to improve the carfollowing model while the data only contain loop detector data [11-14]. Additionally, to calibrate the parameters of car-following models, researchers also focused on the time headway and spatial headway distributions of car-following models $[15,16]$ and phase diagrams $[17,18]$.

The second kind of study learns a new car-following model from the historical trajectory data based on the machine learning methods. Generally, the methods input features such as velocities, velocity differences, and position differences; then, the velocity and acceleration in the next time interval are output [19-21]. The data-driven methods could reduce the human interference and better fit the complex driving behavior observed.

2.2. Car-Following Model of CAVs. Recently, several researchers focused on the car-following model of CAVs. Along with the future introduced CAV technology, the CAV car-following model should be proposed to simulate the future traffic flow, whereas CAVs have different car-following features from HDVs because CAVs can obtain the traffic condition information of the entire network.

Treiber et al. first developed the intelligent driver model (IDM) [22]. The IDM is commonly used in intelligentvehicle situation because it can output the realistic acceleration on a single lane. Through adjusting the parameters, the revised IDM can be applied into CAV simulation [23]. Yet, the deceleration of IDM could be unrealistically high while the current spatial headway is significantly smaller than the expected spatial headway. Thus, Treiber et al. considered that the current spatial headway is smaller than the expected spatial headway as a relatively mild-critical condition [24]. To solve the problem, Kesting et al. reduced the unrealistic deceleration by combining the IDM and the constant acceleration heuristics $(\mathrm{CAH})$, and the adaptive cruise control (ACC) model was proposed [25]. Next, Zhou et al. improved the cooperative intelligent demand model based on the IDM and tested the CAV system performance [23].

\subsection{Bounded Rationality Applied in the Car-Following Model.}

In traffic flow models, bounded rationality is essential for simulating HDVs' driving behaviors more realistic, whereas the above models did not consider bounded rationality [26].

To consider the bounded rationality in the car-following model, Tang et al. [27-30] took bounded rationality into account and developed two car-following models to study the driving behavior and vehicles' emissions. Di et al. [31] stimulated the impacts of bounded rationality on the drivers' route choice behavior. Then, Li et al. $[32,33]$ explored the relationship between bounded rationality and commuting behaviors. Jiang and Deng [34] proposed a Bayesian network to describe travel time distribution based on bounded rationality. In the frame of the nonlinear pairwise swapping rule, Zhang et al. [35] used absolute bounded rationality and relatively bounded rationality to study the route choice behaviors of drivers. Guo et al. [36] proposed a dynamic pricing policy based on bounded rationality and studied the impacts of the policy on traffic system optimization. Li et al. [37] developed a Markov Logic Network method to combine the bounded rationality principle with traffic choice and quantify characteristics and choice models of travelers. Batista et al. [38] used a Lighthill-Whitham-Richards mesoscopic traffic simulator to explore the impacts of bounded rationality on a traffic network performance. Xue et al. [39] explored the impacts of bounded rationality on commuter departure time choice. Then, Fujino and Chen [40] incorporated the bounded rationality of drivers into the traffic network and studied the performance of the traffic network. Moreover, Tang et al. [27-30, 41] and Leng et al. $[42,43]$ developed a car-following model to study vehicles' trip costs in different situations. Then, Tang et al. [44], respectively, proposed two feedback strategies and studied each vehicle's trip cost under the feedback strategies. Based on the research of Tang et al. [44], Ou et al. [10] incorporated bounded rationality into two feedback strategies to study each vehicle's trip cost under different feedback strategies.

The researchers above, respectively, focused on the carfollowing models based on HDVs and CAVs, and they introduced the bounded rationality into HDVs' carfollowing models to simulate the driving behaviors more realistic. However, they did not study the traffic flow model considering the mixed traffic conditions of HDVs and CAVs. Furthermore, they did not consider the randomness of HDVs' drivers' bounded rationality. To bridge these research gaps, the random bounded rationality is introduced into the car-following model of HDVs and study the impact 
of random bounded rationality on the mixed traffic conditions of HDVs and CAVs.

\section{Data}

3.1. Data Collection. This research aims to study the status of mixed traffic flow between HDVs and CAVs, and the random bounded rationality of HDVs should be considered. To find out the distribution laws of drivers' bounded rationality, we did some investigations about the bounded rationality of HDVs in April 2020.

For investigating the bounded rationality of HDVs, we designed a questionnaire. The detailed questionnaire design is shown in Table 1.

We choose respondents from different ages and different genders to do a questionnaire survey through by stratified sampling method (see Table 2). Then, we delete the wrong questionnaires, such as incomplete questionnaires, and questionnaires containing error information. The survey contains 1,000 questionnaires, including 979 valid questionnaires. The purpose of the questionnaire is to investigate the $\varepsilon_{n}\left(\varepsilon_{n}\right.$ is a parameter which reflects the $n$th driver's bounded rationality) and $v_{A B}\left(v_{A B}\right.$ is a parameter that reflects the tolerance of speed difference between the route $a$ and route $b$, where the lengths are, respectively, $L_{a}$ and $L_{b}$, and the routes are as for Figure 1). Referring to the research of Ou et al. [10], reference values of $\varepsilon_{n}$ and $v_{A B}$ are 0.1 and 2 (see Table 2). To test the internal consistency reliability of the questionnaire, we use SPSS to calculate the value of Cronbach's alpha (see Table 2). The value of Cronbach's alpha indicates that the questionnaire is reasonable.

3.2. Data Processing. In this paper, we investigated $\varepsilon_{n}$ and $v_{A B}$ of drivers, and we used ORIGIN to fit the data. Statistical results and the fitting curves are shown in Figures 1 and 2 . Moreover, fitting parameters of $\varepsilon_{n}$ and $v_{A B}$ are shown in Table 3.

Figures 2 and 3 show the fitting condition of $\varepsilon_{n}$ and $v_{A B}$. Additionally, $R^{2}$ is the goodness of fit, which could represent the fitting degree of data. $R^{2}$ of $\varepsilon_{n}$ and $v_{A B}$ demonstrate that they follow the normal distribution, and their distribution is as follows:

$$
\begin{aligned}
\varepsilon_{n} & \sim N\left(0.101,0.021^{2}\right), \\
v_{A B} & \sim N\left(1.897,0.280^{2}\right) .
\end{aligned}
$$

\section{Methods}

4.1. Car-Following Model of HDVs. The basic car-following model is as follows:

$$
\frac{\mathrm{d} v_{n}}{\mathrm{~d} t}=f\left(v_{n}, \Delta x_{n}, \Delta v_{n}, \ldots\right),
$$

where $v_{n}, \Delta x_{n}$, and $\Delta v_{n}$ are, respectively, the $n$th vehicle's velocity, headway, and relative speed; $f$ is the stimulus function.
We can obtain different car-following models from equation (2), e.g., the optimal velocity (OV) model, the generalized force (GF) model, and the full velocity difference (FVD) model [45]. Comparing with the OV model and GF model, the FVD model can produce the desired result and disappear unrealistically high acceleration [42]. Since the model could simulate many complex traffic phenomena, we use the FVD model to simulate vehicles' behavior. The FVD model [45] is as follows:

$$
\frac{\mathrm{d} v_{n}}{\mathrm{~d} t}=k\left(V\left(\Delta x_{n}\right)-v_{n}\right)+\gamma \Delta v_{n}
$$

where $k$ and $\gamma$ are two reactive coefficients; $V$ is the optimal velocity; $\Delta x_{n}$ and $\Delta v_{n}$ are, respectively, the $n$th headway and relative speed. Jiang et al. [45] defined $k, \gamma$, and $V\left(\Delta x_{n}\right)$ as

$$
\begin{aligned}
k & =0.41, \\
\gamma & = \begin{cases}0.5, & \text { if } \Delta x_{n}<100, \\
0, & \text { otherwise, }\end{cases} \\
V\left(\Delta x_{n}\right) & =V_{1}+V_{2} \tanh \left(C_{1}\left(\Delta x_{n}-l_{c}\right)-C_{2}\right),
\end{aligned}
$$

where $l_{c}$ is the vehicles' average length; $V_{1}, V_{2}, C_{1}, C_{2}$, and $l_{c}$ are parameters which were calibrated by Helbing and Tilch [46], and they indicated optimal parameter values. $V_{1}, V_{2}$, $C_{1}, C_{2}$, and $l_{c}$ are defined as [45]

$$
\begin{aligned}
V_{1} & =6.75 \mathrm{~m} / \mathrm{s}, \\
V_{2} & =7.91 \mathrm{~m} / \mathrm{s}, \\
C_{1} & =0.13 \mathrm{~m}^{-1}, \\
C_{2} & =1.57, \\
l_{c} & =5 \mathrm{~m} .
\end{aligned}
$$

To study the impacts of bounded rationality, Tang et al. [27] proposed a car-following model with bounded rationality as

$\frac{\mathrm{d} v_{n}}{\mathrm{~d} t}= \begin{cases}0, & \text { if }\left|k\left(V\left(\Delta x_{n}\right)-v_{n}\right)+\gamma \Delta x_{n}\right| \leq \varepsilon_{n}, \\ k\left(V\left(\Delta x_{n}\right)-v_{n}\right)+\gamma \Delta x_{n}, & \text { otherwise, }\end{cases}$

where $\varepsilon_{n}$ is a parameter that reflects the $n$th driver's bounded rationality; $k$ and $\gamma$ are two reactive coefficients; $v_{n}, \Delta x_{n}$ and $\Delta v_{n}$ are, respectively, the $n$th vehicle's velocity, headway, and relative speed; $V$ is the optimal velocity; and $\mathrm{d} v_{n} / \mathrm{d} t$ indicates the $n$th vehicle's accelerated velocity.

4.2. Car-Following Model of CAVs. The PATH Laboratory of the University of California, Berkeley, has carried out longterm research on the cooperative adaptive cruise control (CACC) model and proposed a CACC car-following model based on a constant headway [47-50]. The model structure is as 
TABLE 1: The detailed questionnaire design.

\begin{tabular}{lc}
\hline Questions & Answers \\
\hline What is your gender? & Male/female \\
What is your age? & - \\
Where is the registration location of your vehicle? & Reference values are in $(0,0.2)$ \\
While you drive a car following the forehead vehicle, what is your reaction & \\
speed of changing your velocity? & Reference values are in $(1,3)$ \\
While you choose the route in a two-route traffic network, what is your tolerance of & \\
speed difference between the two routes?
\end{tabular}

TABle 2: Data structure description.

\begin{tabular}{lc}
\hline Indicators & Description (Cronbach's $\alpha=0.874)$ \\
\hline Age & Age of drivers. 250 drivers aged 20-30, 30-40, 40-50, and 50-60 were selected \\
Gender & Gender; 1: male, 2: female. Respondents of all ages included half females and half males \\
$\varepsilon_{n}$ & The reaction speed of drivers. Range in $(0,0.2)$ \\
$v_{A B}(\mathrm{~m} / \mathrm{s})$ & Tolerance of speed difference between the two routes. Range in $(1,3)$ \\
\hline
\end{tabular}

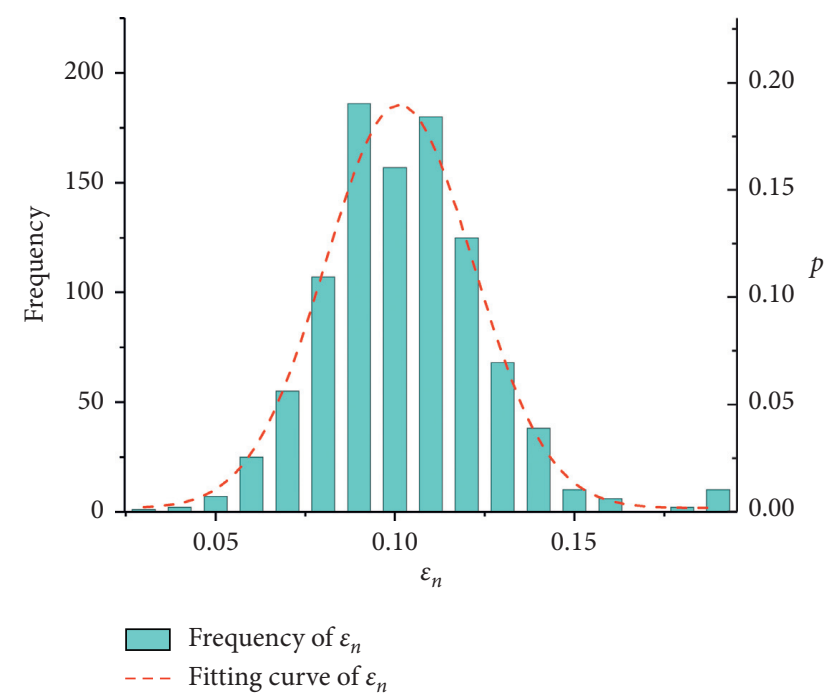

Figure 1: Statistical result and the fitting curves of $\varepsilon_{n}$.

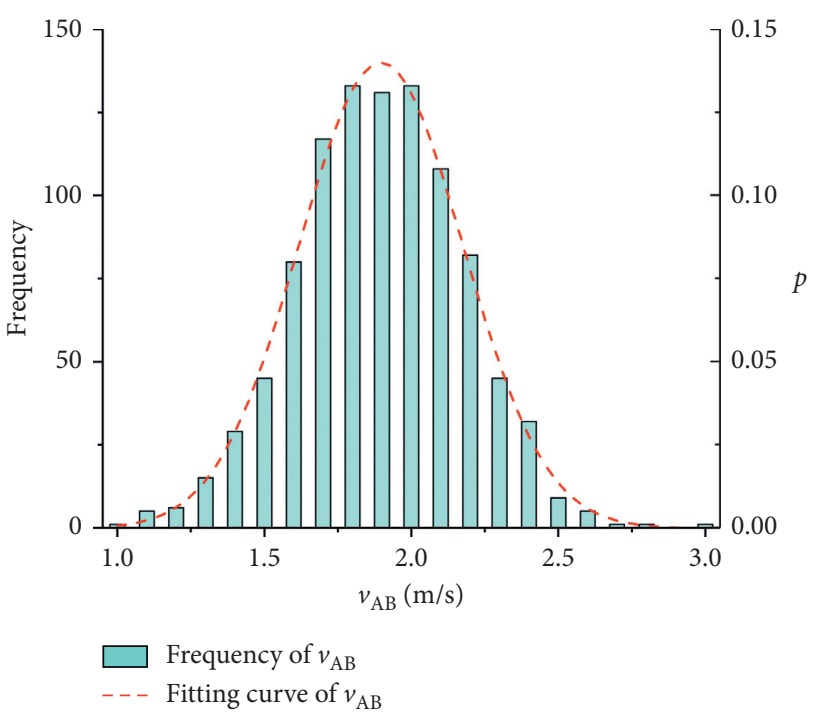

FIgURE 2: Statistical result and the fitting curves of $v_{A B}$.
TABLE 3: Fitting parameters of $\varepsilon_{n}$ and $v_{A B}$.

\begin{tabular}{lccccc}
\hline & Minimum & Maximum & $\begin{array}{l}\text { Mean } \\
\text { value }\end{array}$ & $\begin{array}{c}\text { Standard } \\
\text { deviation }\end{array}$ & $R^{2}$ \\
\hline$\varepsilon_{n}$ & 0.03 & 0.19 & 0.101 & 0.021 & 0.908 \\
$v_{A B}$ & 1.0 & 3.0 & 1.897 & 0.280 & 0.913 \\
$(\mathrm{~m} / \mathrm{s})$ & & & & \\
\hline
\end{tabular}

$$
\begin{aligned}
a_{n}(t)= & k_{1} a_{n-1}(t)+k_{2}\left[x_{n-1}(t)-x_{n}(t)-t_{g} v_{n}(t)-l-s_{0}\right] \\
& +k_{3}\left[v_{n-1}(t)-v_{n}(t)\right]
\end{aligned}
$$

where $a_{n}(t)$ represents the acceleration of vehicle $n$ at time $t$; $x_{n}(t)$ indicates the location of vehicle $n$ at time $t ; v_{n}(t)$ is the velocity of vehicle $n$ at time $t ; l$ represents the length of the vehicle; $s_{0}$ indicates the safe distance between vehicles; $t_{g}$ is the expected time headway of CACC; $k_{1}, k_{2}$, and $k_{3}$ are coefficients of the model, and we take $k_{1}, k_{2}$, and $k_{3}$ as 1.0, 0.2 , and 3.0, respectively [51].

The CACC model proposed by PATH is clear in the physical meaning and is currently commonly used as the CACC car-following model. However, the expected spatial headway under the constant spatial headway carfollowing strategy is a linear function of speed, which makes the impact of traffic flow characteristics with different spatial headway different. Therefore, this paper proposes the nonlinear dynamics-expected time headway strategy of the CACC car-following model. Based on the traffic flow following characteristics, the lower vehicle speed makes expected spatial headway smaller. Based on the results of [52], the dynamic expected spatial headway is as

$$
h_{d}(t)=\frac{l+s_{0}}{1-\left(v_{n}(t) / v_{0}\right)},
$$

where $h_{d}(t)$ represents the expected spatial headway; $v_{0}$ is the velocity of free traffic flow. According to [53], $s_{0}$ and $l$ are, respectively, $2 \mathrm{~m}$ and $5 \mathrm{~m}$. 


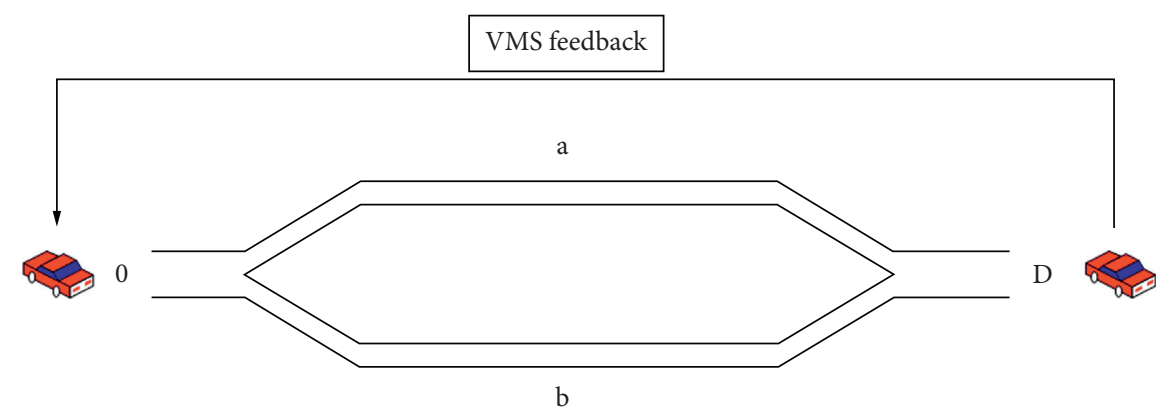

FIgURE 3: The structure of the two-route traffic network.

4.3. Proposed Feedback Strategy. Next, we propose a feedback strategy in the traffic network. As for the feedback strategies, researchers proposed several feedback strategies to study each vehicle's trip cost [54-56], whereas the feedback strategies did not consider random bounded rationality and the penetration rate of CAVs. Therefore, we proposed HFS in a microsimulation, which takes random bounded rationality and penetration rate of CAVs into account. In HFS, because drivers usually choose the route based on the speed of traffic flow, we take the average speed of vehicles on the route as the reference index. Then, we give the following underlying assumptions:

(1) The traffic network includes routes $a$ and $b$ (see Figure 3), where the lengths are, respectively, $L_{a}$ and $L_{b} ; N$ vehicles enter/leave the network at the origin/ destination, where each vehicle's no. on the route $a / b$ is $n_{a} / n_{b}$, and its time headway at the origin is $\Delta t_{0}$; and the routes are homogeneous.

(2) When $n \leq N_{0}$, the $n$ the driver randomly selects route; when $n>N_{0}$, the driver selects a route based on the HFS.

(3) $M_{a / b}$ is the number of vehicles on route $a / b ; v_{j, a / b}$ is the $j$ th vehicle's speed on route $a / b$.

(4) $v_{A B}>0, \varepsilon_{n}>0$ are parameters that represent random bounded rationality.

(5) The HFS with random bounded rationality consists of HVFS (HDV feedback strategy) and CVFS (CAV feedback strategy); $p r_{C A V}$ is the penetration rate of CAVs. In this strategy, the differences between HDVs and CAVs are reflected in $\varepsilon_{n}$ and $v_{A B}$. Therefore, we proposed HVFS and CVFS to reflect the differences in the mobility characteristics of these vehicles.

(6) The HVFS with random bounded rationality is defined as follows:

(i) If $\left(1 / M_{a}\right) \sum_{j=1}^{M_{a}} v_{j, a}>\left(1 / M_{b}\right) \sum_{j=1}^{M_{b}} v_{j, b}+v_{A B}$, the vehicle selects route $a$

(ii) If $\left(1 / M_{b}\right) \sum_{j=1}^{M_{b}} v_{j, b}>\left(1 / M_{a}\right) \sum_{j=1}^{M_{a}} v_{j, a}+v_{A B}$, the vehicle chooses route $b$ (iii) If $\quad\left|\left(1 / M_{a}\right) \sum_{j=1}^{M_{a}} v_{j, a}-\left(1 / M_{b}\right) \sum_{j=1}^{M_{b}} v_{j, b}\right| \leq v_{A B}$, the vehicle randomly chooses route

(7) The CVFS is defined as follows:

(i) If $\left(1 / M_{a}\right) \sum_{j=1}^{M_{a}} v_{j, a} \geq\left(1 / M_{b}\right) \sum_{j=1}^{M_{b}} v_{j, b}$, the vehicle selects route $a$

(ii) If $\left(1 / M_{a}\right) \sum_{j=1}^{M_{a}} v_{j, a}<\left(1 / M_{b}\right) \sum_{j=1}^{M_{b}} v_{j, b}$, the vehicle chooses route $b$

Finally, we define the calculation of trip cost as

$$
\begin{gathered}
T_{n}=\alpha \Delta t_{n}, \\
T_{a}=\sum_{k=1}^{M_{a}} T_{k, a}, \\
T_{b}=\sum_{k=1}^{M_{b}} T_{k, b}, \\
T_{\text {total }}=T_{a}+T_{b},
\end{gathered}
$$

where $T_{n}$ is the $n$th vehicle's trip cost; $t_{n}$ is the $n$th vehicle's travel time; $\alpha$ is the value of time (the unit is Yuan/s); $T_{a}, T_{b}$ is the total trip cost on the route $a / b$, respectively; $T_{k, a}, T_{k, b}$ is the $k$ th vehicle's trip cost on the route $a / b$, respectively; and $T_{\text {total }}$ is the traffic network's total trip cost. In the numerical test, parameters [27, 44] are as equation (14). The numerical test indicates that the first ten vehicles randomly choose route, and then, other vehicles choose the route based on HFS. Moreover, we assume that the time value is $0.01 \mathrm{Yuan} / \mathrm{s}[27,44]$ :

$$
\begin{aligned}
N & =100, \\
N_{0} & =10, \\
L_{a} & =L_{b}=1000, \\
\alpha & =0.01 \text { Yuan } / \mathrm{s}, \\
\Delta t & =0.1 .
\end{aligned}
$$

We use the numerical test to study the impacts of random bounded rationality, and penetration rate of CAVs 
on vehicles' trip cost under HFS in the traffic network, and we use the Euler difference to discretize equations (7) to (15) and (16):

$$
\begin{aligned}
& v_{n}(t+\Delta t)=v_{n}(t)+\frac{\mathrm{d} v_{n}(t)}{\mathrm{d} t} \cdot \Delta t, \\
& x_{n}(t+\Delta t)=x_{n}(t)+v_{n}(t) \cdot \Delta t+\frac{1}{2} \frac{\mathrm{d} v_{n}(t)}{\mathrm{d} t} \cdot(\Delta t)^{2},
\end{aligned}
$$

where $\Delta t=0.1 \mathrm{~s}$ is the time-step length and $x_{n}$ is the $n$th vehicle's position.

\section{Results}

First, we study the effects of CAV penetration rate on vehicle trip costs under the HFS (see Figures 4-6). In the HFS, we not only considered the random bounded rationality but also took the penetration rate of CAVs into account. In the $\mathrm{HFS}, \varepsilon_{n}$ and $v_{A B}$ indicate the random bounded rationality, and $p r_{C A V}$ represents the penetration rate of CAVs. And in the numerical test, the value range of $\Delta t_{0}$ is in $(0,6)$ and the value range of $p r_{C A V}$ is in $(0,1)$. From Figures $4-6$, we can obtain the following findings:

(1) When $p r_{C A V}$ is relatively low (e.g., $p r_{C A V}=0.25$ ), the difference between the travel time of two routes is prominent. As $p r_{C A V}$ rises, the difference between the travel time of vehicles on the two routes drops.

(2) When $p r_{C A V}$ is relatively high (e.g., $p r_{C A V}=0.75$ ), the difference between the travel time of two routes is inconspicuous.

(3) When $\Delta t_{0}$ is short (e.g., $\Delta t_{0}=0.5 \mathrm{~s}$ ), vehicles' travel time rises with its number; each vehicle' travel time on each route is slightly longer than the corresponding one in the study [10], but each vehicle's travel time in our study is fluctuant. The result shows that random bounded rationality could increase the volatility of each vehicle's travel time.

(4) When $\Delta t_{0}$ is relatively long (e.g., $\Delta t_{0}=1.5 \mathrm{~s}, 2.5 \mathrm{~s}$ ), each vehicle's travel time approximately equals to a constant with fluctuation. In addition, as the $\Delta t_{0}$ grows, the fluctuation of each vehicle's trip cost decreases.

(5) In summary, vehicles' travel time reduced with $\Delta t_{0}$ and $p r_{C A V}$, and random bounded rationality slightly increases the travel time of vehicles and raises the volatility of vehicles.

Then, we analyze the effects of random bounded rationality and the penetration rate of CAVs on the total trip cost of the traffic network (see Figures 7-9).

Figures 7 and 8 show that the total trip cost drops rapidly while $\Delta t_{0}<1.5 \mathrm{~s}$, because of each vehicle's travel time on

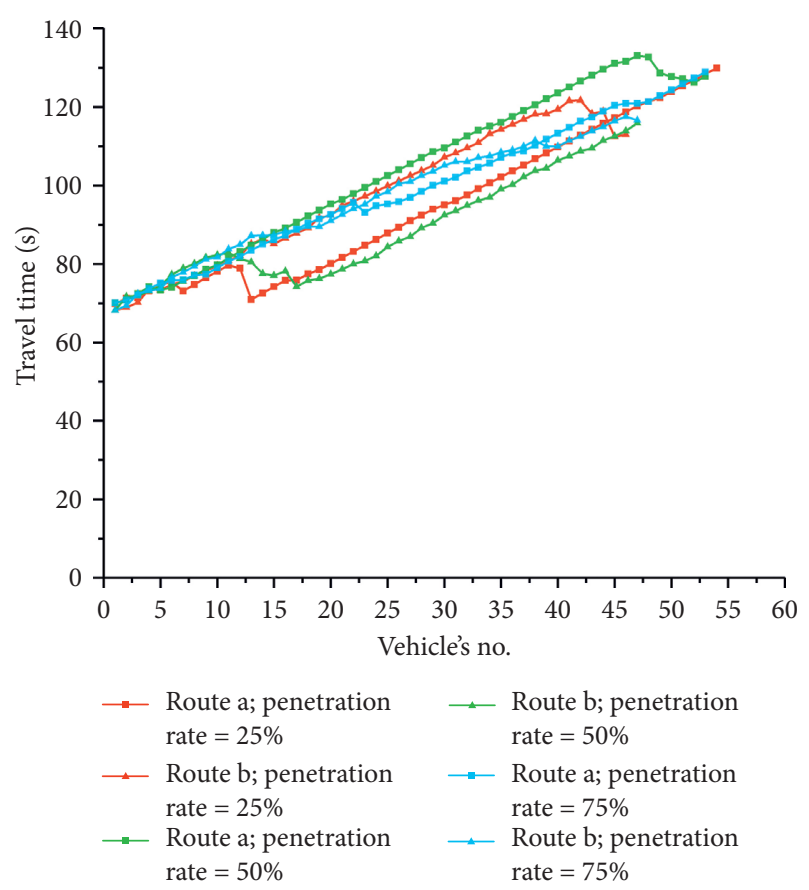

FIgURE 4: Each vehicle's travel time under HFS while $\Delta t_{0}=0.5 \mathrm{~s}$.

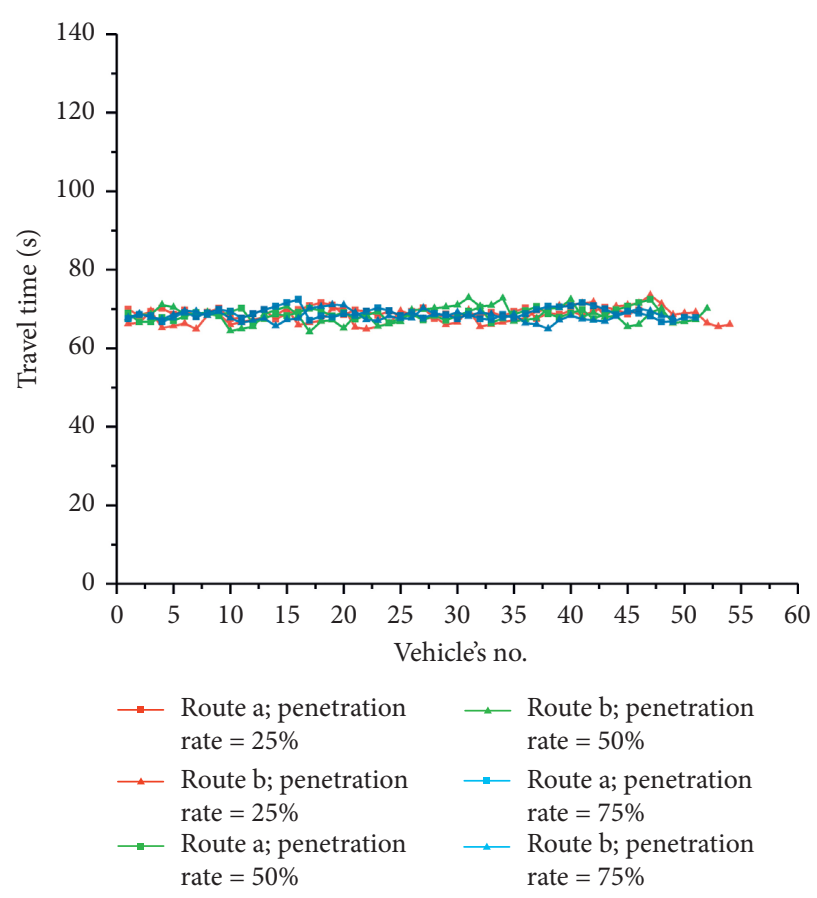

FIgURE 5: Each vehicle's travel time under HFS while $\Delta t_{0}=1.5 \mathrm{~s}$.

each route decreases rapidly with $\Delta t_{0}$. Moreover, when $\Delta t_{0} \geq 1.5 \mathrm{~s}$, the total trip cost smoothly decreases. Furthermore, as the penetration rate of CAVs grows, the total trip 


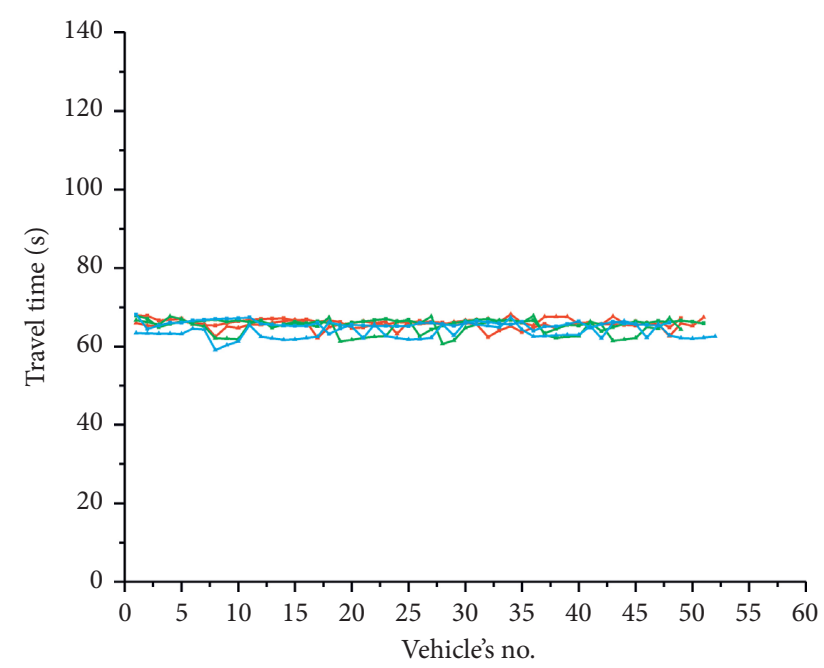

\begin{tabular}{|c|c|}
\hline $\begin{array}{l}\text { Route a; penetration } \\
\text { rate }=25 \%\end{array}$ & $\begin{array}{l}\longrightarrow \text { Route b; penetration } \\
\text { rate }=50 \%\end{array}$ \\
\hline $\begin{array}{l}\text { Route } b ; \text { penetration } \\
\text { rate }=25 \%\end{array}$ & $\begin{array}{l}- \text { Route a; penetration } \\
\text { rate }=75 \%\end{array}$ \\
\hline $\begin{array}{l}\text { Route a; penetration } \\
\text { rate }=50 \%\end{array}$ & $\begin{array}{l}\triangle \quad \text { Route b; penetration } \\
\text { rate }=75 \%\end{array}$ \\
\hline
\end{tabular}

Figure 6: Each vehicle's travel time under HFS while $\Delta t_{0}=2.5 \mathrm{~s}$.

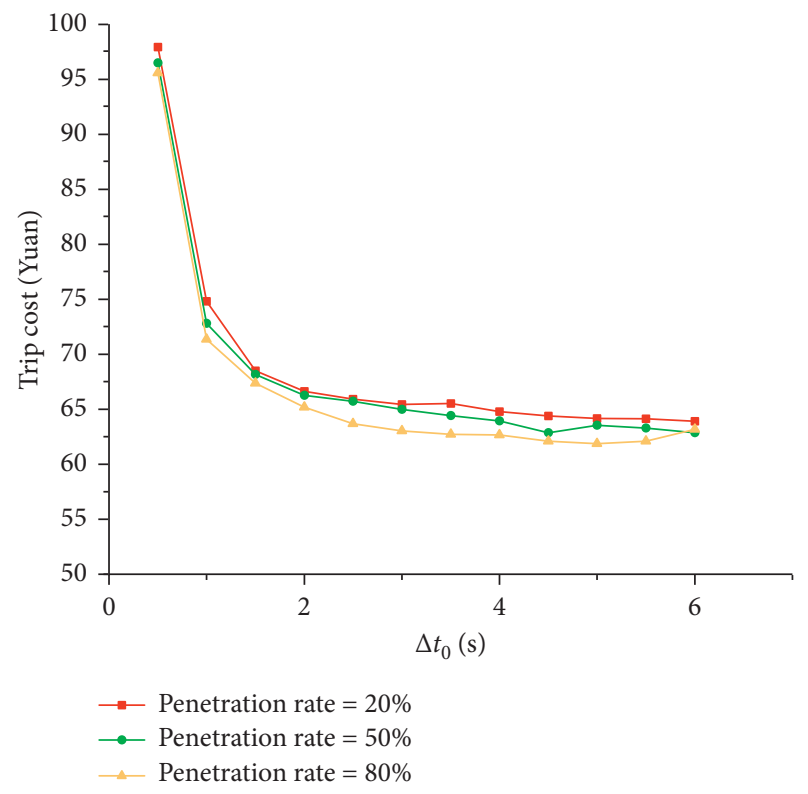

Figure 7: The total trip cost under HFS while $\operatorname{pr}_{C A V}=0.2,0.5$, and 0.8 .

cost goes down consistently. It indicates that the penetration rate of CAVs effectively reduces the total trip cost in the traffic network. Impacts of $\Delta t_{0}$ and $p r_{C A V}$ are shown in Figure 9, and higher $p r_{C A V}$ and longer $\Delta t_{0}$ lead to less total trip cost (see Figure 9).

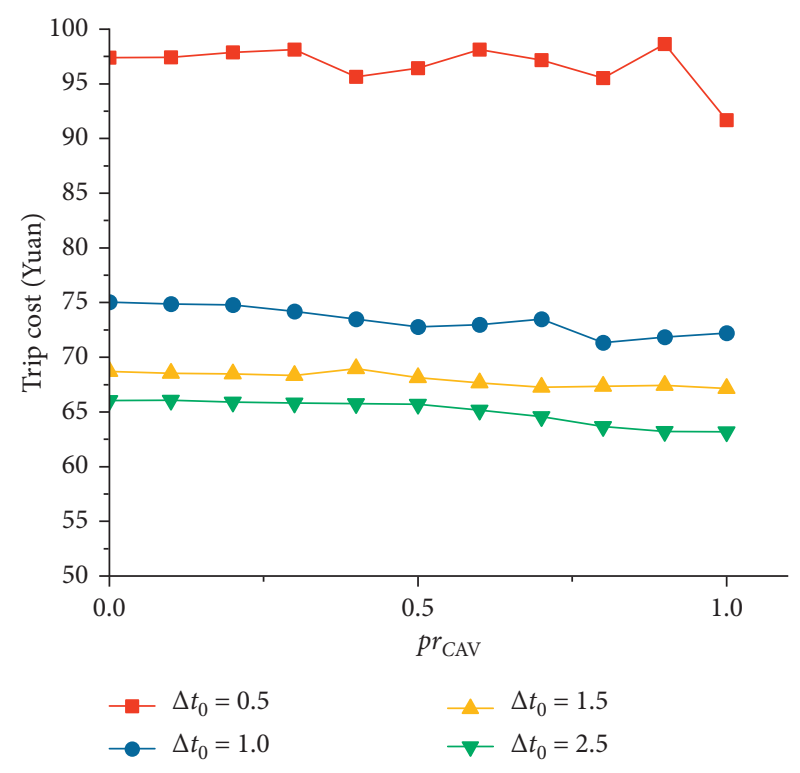

Figure 8: The total trip cost under HFS while $\Delta t_{0}=0.5 \mathrm{~s}, 1.0 \mathrm{~s}, 1.5 \mathrm{~s}$, and $2.5 \mathrm{~s}$.

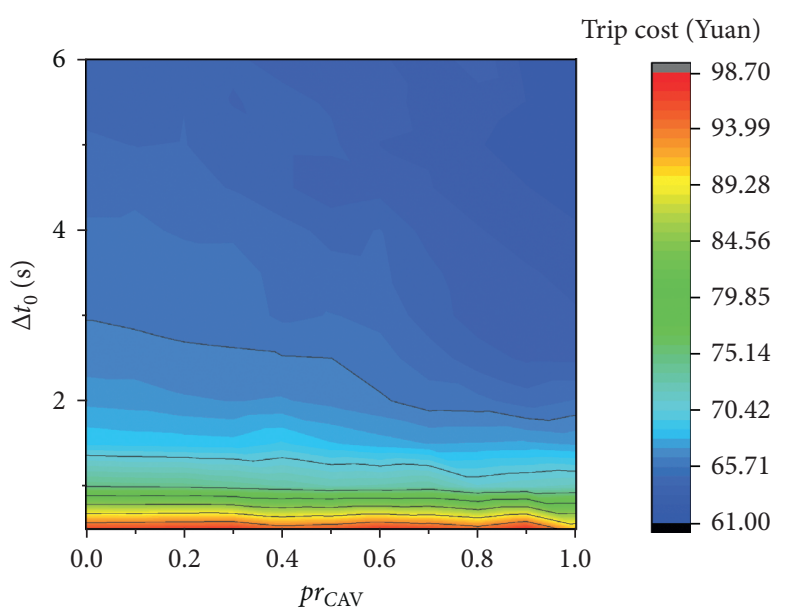

Figure 9: Impacts of $\Delta t_{0}$ and $p r_{C A V}$ on system total trip cost.

\section{Discussion and Conclusions}

6.1. Discussion. In this paper, we studied the effects of the CAV penetration rate on each vehicle's travel time and system total trip cost under the HFS considered the random bounded rationality, and we can obtain the following findings:

(1) The bounded rationality of drivers follows the normal distribution, and the distribution is as equation (1). Before the investigation, we give the interviewees a reference value. It may influence their choices of bounded rationality. 
(2) The CAV penetration rate has qualitative effects on the travel time of each vehicle and system total trip cost (see Figures 7-9). As $p r_{C A V}$ increases, the travel time of each vehicle and total trip cost both smoothly decrease because of the CAVs' entry reduces the average hesitate time of all vehicles.

(3) The total trip cost of the traffic network rapidly drops when $\Delta t_{0}<1.5 \mathrm{~s}$, because of each vehicle's travel time on each route declines rapidly with $\Delta t_{0}$. Moreover, when $\Delta t_{0} \geq 1.5 \mathrm{~s}$, the system total cost smoothly decreases. And Figure 9 indicates that larger $\Delta t_{0}$ leads to less travel time and total trip cost.

Nevertheless, this research still has some limitations. For example, we did not test the numerical results by experiments on the road and a real medium-sized road network should be studied. Moreover, we will take more factors into account to simulate the traffic flow realistically, such as CAV market penetration, platooning intensity effects, single lane, and managed lane.

6.2. Conclusions. Many feedback strategies were proposed to study the condition of the traffic flow in traffic networks [57], but the feedback strategies do not consider the randomness of bounded rationality and the penetration rate of CAVs in the mixed traffic flow of HDVs and CAVs. In this paper, we proposed HFS, which takes random bounded rationality and the penetration rate of CAVs into account. According to the numerical results, we found that drivers' bounded rationality follows the normal distribution $\left(\varepsilon_{n} \sim N(0.101\right.$, $\left.\left.0.021^{2}\right), v_{A B} \sim N\left(1.897,0.280^{2}\right)\right)$. Based on the distribution of random bounded rationality, we found that the penetration rate of CAVs could effectively reduce the system total trip cost. Furthermore, $\Delta t_{0}$ under HFS has qualitative effects on the trip cost of vehicles.

In the future, more experiments can be concerned. We can test the numerical results by experiments on the road and simulate the traffic flow in a real medium-sized road network. Furthermore, we will take more factors into account to simulate the traffic flow realistically, such as CAV market penetration, platooning intensity effects, single lane, and managed lane, to make more contributions to the CAV study.

\section{Data Availability}

The data used to support the findings of this study are available from the corresponding author upon request.

\section{Conflicts of Interest}

The authors declare that there are no conflicts of interest regarding the publication of this paper.

\section{Acknowledgments}

This study was supported by the Natural Science Foundation of Zhejiang Province (grant no. LQ19E080003), Philosophy and Social Science Program of Ningbo (grant no. G20-ZX37), and National Natural Science Foundation of China (grant no. 52002282).

\section{References}

[1] X. Li, W. Wang, C. Xu, Z. Li, and B. Wang, "Multi-objective optimization of urban bus network using cumulative prospect theory," Journal of Systems Science and Complexity, vol. 28, no. 3, pp. 661-678, 2015.

[2] X. Li, J. Tang, X. Hu, and W. Wang, "Assessing intercity multimodal choice behavior in a Touristy city: a factor analysis," Journal of Transport Geography, vol. 86, 2020.

[3] G. H. Peng and D. H. Sun, "A dynamical model of car-following with the consideration of the multiple information of preceding cars," Physics Letters A, vol. 374, no. 15-16, pp. 1694-1698, 2010.

[4] G. H. Peng, W. Song, Y. J. Peng, and S. H. Wang, "A novel macro model of traffic flow with the consideration of anticipation optimal velocity," Physica A: Statistical Mechanics and Its Applications, vol. 398, pp. 76-82, 2014.

[5] Z. Liu, H. Chen, Y. Li, and Q. Zhang, "Taxi demand prediction based on a combination forecasting model in hotspots," Journal of Advanced Transportation, vol. 2020, Article ID 1302586, 13 pages, 2020.

[6] S. Yu and Z. Shi, "Analysis of car-following behaviors considering the green signal countdown device," Nonlinear Dynamics, vol. 82, no. 1-2, pp. 731-740, 2015.

[7] X. Wu, X. Zhao, H. Song, Q. Xin, and S. Yu, "Effects of the prevision relative velocity on traffic dynamics in the ACC strategy," Physica A: Statistical Mechanics and Its Applications, vol. 515, pp. 192-198, 2019.

[8] X. Li, T. Yang, J. Liu, X. Qin, and S. Yu, "Effects of vehicle gap changes on fuel economy and emission performance of the traffic flow in the ACC strategy," PLoS One, vol. 13, no. 7, 2018.

[9] H.-X. Ge, P.-J. Zheng, S.-M. Lo, and R.-J. Cheng, "TDGL equation in lattice hydrodynamic model considering driver's physical delay," Nonlinear Dynamics, vol. 76, no. 1, pp. 441-445, 2013.

[10] H. Ou, T.-Q. Tang, and J. Zhang, "Impacts of bounded rationality on trip cost in a two-route traffic network," Physica A: Statistical Mechanics and Its Applications, vol. 540, 2020.

[11] E. Brockfeld, R. D. Kühne, and P. Wagner, "Calibration and validation of microscopic traffic flow models," Transportation Research Record: Journal of the Transportation Research Board, vol. 1876, no. 1, pp. 62-70, 2004.

[12] E. Brockfeld, R. D. Kühne, and P. Wagner, "Calibration and validation of microscopic models of traffic flow," Transportation Research Record: Journal of the Transportation Research Board, vol. 1934, no. 1, pp. 179-187, 2005.

[13] H. Rakha, C. C. Pecker, and H. B. B. Cybis, "Calibration procedure for Gipps car-following model," Transportation Research Record: Journal of the Transportation Research Board, vol. 1999, no. 1, pp. 115-127, 2007.

[14] H. Rakha and W. Wang, "Procedure for calibrating Gipps carfollowing model," Transportation Research Record: Journal of the Transportation Research Board, vol. 2124, no. 1, pp. 113124, 2009.

[15] X. Jin, Y. Zhang, F. Wang et al., "Departure headways at signalized intersections: a log-normal distribution model approach," Transportation Research Part C: Emerging Technologies, vol. 17, no. 3, pp. 318-327, 2009.

[16] L. Li and X. Chen, "Vehicle headway modeling and its inferences in macroscopic/microscopic traffic flow theory: a 
survey," Transportation Research Part C: Emerging Technologies, vol. 76, pp. 170-188, 2017.

[17] M. Treiber and A. Kesting, "Validation of traffic flow models with respect to the spatiotemporal evolution of congested traffic patterns," Transportation Research Part C: Emerging Technologies, vol. 21, no. 1, pp. 31-41, 2012.

[18] X. Chen, L. Li, and Z. Li, "Phase diagram analysis based on a temporal-spatial queueing model," IEEE Transactions on Intelligent Transportation Systems, vol. 13, no. 4, pp. 17051716, 2012.

[19] A. Khodayari, A. Ghaffari, R. Kazemi, F. Alimardani, and R. Braunstingl, "Improved adaptive neuro fuzzy inference system car-following behaviour model based on the drivervehicle delay," IET Intelligent Transport Systems, vol. 8, no. 4, pp. 323-332, 2013.

[20] L. Chong, M. M. Abbas, A. Medina Flintsch, and B. Higgs, "A rule-based neural network approach to model driver naturalistic behavior in traffic," Transportation Research Part C: Emerging Technologies, vol. 32, pp. 207-223, 2013.

[21] D. Yang, L. Zhu, Y. Liu, D. Wu, and B. Ran, "A novel carfollowing control model combining machine learning and kinematics models for automated vehicles," IEEE Transactions on Intelligent Transportation Systems, vol. 20, no. 6, pp. 1991-2000, 2019.

[22] M. Treiber, A. Hennecke, and D. Helbing, "Congested traffic states in empirical observations and microscopic simulations," Physical Review E, vol. 62, no. 2, pp. 1805-1824, 2000.

[23] M. Zhou, X. Qu, and S. Jin, "On the impact of cooperative autonomous vehicles in improving freeway merging:a modified intelligent driver model-based approach," IEEE Transactions on Inteligent Transportation Systems, vol. 18, no. 6, pp. 1422-1428, 2017.

[24] M. Treiber, A. Kesting, and D. Helbing, "Understanding widely scattered traffic flows, the capacity drop, and platoons as effects of variance-driven time gaps," Physical Review E: Statistical, Nonlinear, and Soft Matter Physics, vol. 74, no. 1, Article ID 016123, 2006.

[25] A. Kesting, M. Treiber, and D. Helbing, "Enhanced intelligent driver model to access the impact of driving strategies on traffic capacity," Philosophical Transactions of the Royal Society A: Mathematical, Physical and Engineering Sciences, vol. 368, no. 1928, pp. 4585-4605, 2010.

[26] W. Wang, H. Sun, Z. Wang, and J. Wu, “Optimal transit fare in a bimodal network under demand uncertainty and bounded rationality," Journal of Advanced Transportation, vol. 48, no. 8, pp. 957-973, 2014.

[27] T.-Q. Tang, H.-J. Huang, and H.-Y. Shang, "Influences of the driver's bounded rationality on micro driving behavior, fuel consumption and emissions," Transportation Research Part D: Transport and Environment, vol. 41, pp. 423-432, 2015.

[28] T.-Q. Tang, H.-J. Huang, and H.-Y. Shang, "An extended macro traffic flow model accounting for the driver's bounded rationality and numerical tests," Physica A: Statistical Mechanics and Its Applications, vol. 468, pp. 322-333, 2017.

[29] T.-Q. Tang, X.-F. Luo, and K. Liu, "Impacts of the driver's bounded rationality on the traffic running cost under the carfollowing model," Physica A: Statistical Mechanics and Its Applications, vol. 457, pp. 316-321, 2016.

[30] T.-Q. Tang, J. Zhang, and K. Liu, “A speed guidance model accounting for the driver's bounded rationality at a signalized intersection," Physica A: Statistical Mechanics and Its Applications, vol. 473, pp. 45-52, 2017.
[31] X. Di, H. X. Liu, and X. Ban, "Second best toll pricing within the framework of bounded rationality," Transportation Research Part B: Methodological, vol. 83, pp. 74-90, 2016.

[32] C.-Y. Li, G.-M. Xu, and T.-Q. Tang, "Social optimum for evening commute in a single-entry traffic corridor with no early departures," Physica A: Statistical Mechanics and Its Applications, vol. 502, pp. 236-247, 2018.

[33] C. Li and H. Huang, "Analysis of bathtub congestion with continuous scheduling preference," Research in Transportation Economics, vol. 75, pp. 45-54, 2019.

[34] X. Jiang and W. Deng, "Considering dynamic knowledge updating in bounded rationality-based route choice modeling," Advances in Mechanical Engineering, vol. 9, no. 9, 2017.

[35] W. Zhang, Z. He, W. Guan, and G. Qi, "Day-to-day rerouting modeling and analysis with absolute and relative bounded rationalities," Transportmetrica A: Transport Science, vol. 14, no. 3, pp. 256-273, 2017.

[36] R.-Y. Guo, H. Yang, H.-J. Huang, and X. Li, "Day-to-day departure time choice under bounded rationality in the bottleneck model," Transportation Research Procedia, vol. 23, pp. 551-570, 2017.

[37] T. Li, H. Guan, J. Ma, G. Zhang, and K. Liang, "Modeling travel mode choice behavior with bounded rationality using Markov Logic Networks," Transportation Letters, vol. 11, no. 6, pp. 303-310, 2017.

[38] S. F. A. Batista, C.-L. Zhao, and L. Leclercq, "Effects of users' bounded rationality on a traffic network performance: a simulation study," Journal of Advanced Transportation, vol. 2018, Article ID 9876598, 20 pages, 2018.

[39] Y. Xue, H. Fan, and H. Guan, "Commuter departure time choice considering parking space shortage and commuter's bounded rationality," Journal of Advanced Transportation, vol. 2019, Article ID 4864287, 7 pages, 2019.

[40] T. Fujino and Y. Chen, "Effects of network structure on the performance of a modeled traffic network under drivers' bounded rationality," Physica A: Statistical Mechanics and Its Applications, vol. 540, 2020.

[41] T.-Q. Tang, T. Wang, L. Chen, and H.-J. Huang, "Analysis of the equilibrium trip cost accounting for the fuel cost in a single-lane traffic system without late arrival," Physica A: Statistical Mechanics and Its Applications, vol. 490, pp. 451457, 2018.

[42] J.-Q. Leng and L. Zhao, “Analysis of electric vehicle's trip cost without late arrival," Physica A: Statistical Mechanics and Its Applications, vol. 469, pp. 761-766, 2017.

[43] J.-Q. Leng, J. Zhai, Q.-W. Li, and L. Zhao, "Construction of road network vulnerability evaluation index based on general travel cost," Physica A: Statistical Mechanics and Its Applications, vol. 493, pp. 421-429, 2018.

[44] T.-Q. Tang, Q. Yu, and K. Liu, "Analysis of the traffic running cost in a two-route system with feedback strategy," Physica A: Statistical Mechanics and Its Applications, vol. 466, pp. 1-9, 2017.

[45] R. Jiang, Q. Wu, and Z. Zhu, "Full velocity difference model for a car-following theory," Physical Review E-American Physical Society, vol. 64, no. 1, 2001.

[46] D. Helbing and B. Tilch, "Generalized force model of traffic dynamics," Physical Review E, vol. 58, no. 1, pp. 133-138, 1998.

[47] S. Shladover, D. Su, and X. Lu, "Impacts ofcooperative adaptive cruise control on freeway traffic flow," Transportation Research Record, vol. 2324, no. 1, pp. 63-70, 2012.

[48] J. Vanderwerfe, S. Shladover, and N. Kourjanskaia, "Modeling effects of driver control assistance systems on traffic," 
Transportation Research Record, vol. 1748, no. 1, pp. 167-174, 2001.

[49] S. E. Shladover and S.-K. Tan, "Analysis of vehicle positioning accuracy requirements for communication-based cooperative collision warning," Journal of Intelligent Transportation Systems, vol. 10, no. 3, pp. 131-140, 2007.

[50] S. E. Shladover, C. A. Desoer, J. K. Hedrick et al., "Automated vehicle control developments in the PATH program," IEEE Transactions on Vehicular Technology, vol. 40, no. 1, pp. 114-130, 1991.

[51] B. Van Arem, C. J. G. Van Driel, and R. Visser, "The impact of cooperative adaptive cruise control on traffic-flow characteristics," IEEE Transactions on Intelligent Transportation Systems, vol. 7, no. 4, pp. 429-436, 2006.

[52] J. Wang and R. Rajamani, "The impact of adaptive cruise control systems on highway safety and traffic flow," Proceedings of the Institution of Mechanical Engineers, Part D: Journal of Automobile Engineering, vol. 218, no. 2, pp. 111130, 2004

[53] Y. Qin, H. Wang, and B. Ran, "Car-following modeling for CACC vehicles and mixed traffic flow analysis," Journal of Transportation Systems Engineering and Information Technology, vol. 18, no. 2, pp. 60-65, 2018.

[54] W.-X. Wang, B.-H. Wang, W.-C. Zheng, C.-Y. Yin, and T. Zhou, "Advanced information feedback in intelligent traffic systems," Physical Review E-American Physical Society, vol. 72, no. 6, 2005.

[55] C.-F. Dong, X. Ma, and B.-H. Wang, "Weighted congestion coefficient feedback in intelligent transportation systems," Physics Letters A, vol. 374, no. 11-12, pp. 1326-1331, 2010.

[56] K. Lee, P. M. Hui, B.-H. Wang, and N. F. Johnson, "Effects of announcing global information in a two-route traffic flow model," Journal of the Physical Society of Japan, vol. 70, no. 12, pp. 3507-3510, 2001.

[57] J.-Y. Zeng, H. Ou, and T.-Q. Tang, "Feedback strategy with delay in a two-route traffic network," Physica A: Statistical Mechanics and Its Applications, vol. 534, 2019. 